\title{
Retraction Note: The microRNA-1246 promotes metastasis in non-small cell lung cancer by targeting cytoplasmic polyadenylation element-binding protein
}

Weihua Huang ${ }^{1}$, Huifen $\mathrm{Li}^{2}$ and Rongcheng Luo ${ }^{1 *}$

\section{Retraction}

This article [1] has been retracted by the Editor. Figure 1 (panels: A, C, D, E, F), 2 (panels: A, E, F, G, H) and 4, as well as parts of the text, were duplicated from Tian et al., 2012 [2]. The findings of this study are therefore unreliable. The Editor has been unable to confirm with Southern Medical University whether an institutional investigation has taken place. We have not been able to contact the authors.

\section{Author details}

'TCM-Integrated Hospital, Southern Medical University, Cancer Center, NO.13 Shiliugang Road, Haizhu District, Guangzhou, Guangdong 510315, China. ${ }^{2}$ Department of Chemotherapy, Zhongshan People's Hospital, Zhongshan, Guangdong 528400, China.

Received: 8 June 2017 Accepted: 26 June 2017

Published online: 18 July 2017

\section{References}

1. Huang W, Li H, Luo R. The microRNA-1246 promotes metastasis in non-small cell lung cancer by targeting cytoplasmic polyadenylation element-binding protein 4. Diagn Pathol. 2015 Jul 25;10:127.

2. Tian Q, Liang L, Ding J, Zha R, Shi H, Wang Q, et al. MicroRNA-550a acts as a pro-metastatic gene and directly targets cytoplasmic polyadenylation element-binding protein 4 in hepatocellular carcinoma. PLoS One. 2012:7(11):e48958.

\footnotetext{
*Correspondence: jackieluorongcheng@163.com

The online version of the original article can be found under doi:10.1186/ s13000-015-0366-1

${ }^{1}$ TCM-Integrated Hospital, Southern Medical University, Cancer Center, NO.13

Shiliugang Road, Haizhu District, Guangzhou, Guangdong 510315, China
} 